\title{
Research Article \\ Effects of Wind on Virtual Plants in Animation
}

\author{
Tina L. M. Derzaph and Howard J. Hamilton \\ Department of Computer Science, University of Regina, Regina, SK, Canada S4S 0A2 \\ Correspondence should be addressed to Howard J. Hamilton; howard.hamilton@uregina.ca
}

Received 1 March 2013; Revised 2 July 2013; Accepted 8 July 2013

Academic Editor: Yiyu Cai

Copyright (C) 2013 T. L. M. Derzaph and H. J. Hamilton. This is an open access article distributed under the Creative Commons Attribution License, which permits unrestricted use, distribution, and reproduction in any medium, provided the original work is properly cited.

\begin{abstract}
This paper presents the Growth-Flow method for animating the effect of wind on the motion and growth of virtual plant branches and leaves. The method incorporates changes to the growth rate when a plant is exposed to winds with speeds higher than a threshold. In particular, growth rate is reduced in branch elongation, increased in the branch radius, reduced in leaf length, and increased in leaf thickness. In addition, when a plant is exposed to wind for long time periods, the branch growth angle is changed to align more closely with the wind vector. The Growth-Flow method incorporates all these effects on growth and motion due to wind in one algorithm.
\end{abstract}

\section{Introduction}

To enhance the realism of virtual plants, the effects of wind on the motion and growth of plants should be modeled. The effects of wind blowing through trees and especially its effects on branches have been extensively studied in computer animation. To date, only a few computer animation researchers have studied the effect of wind on leaf motion, with no consideration of the effects of wind on the growth of virtual plants.

Plants are affected by wind immediately through physical motion and over time through changes in growth. Wind contact with a plant immediately causes physical motion by bending, swaying, or twisting the stem or leaves. Studies on branch movement in trees have approached the issue using physically based methods [1-4], procedurally based methods [5-8], motion capture [9], simulation [10], and shell dynamics [11]. Studies have examined leaf motion with respect to flow [12], biomechanics [13], spring and rotational movement [14], wilting [15], response to wind by broadleaf types [16], response to wind by both broadleaf and needle leaves [14], as a component of overall plant motion [10], and as part of shell space [11]. In addition, animation with leaf growth effects has been performed using a physically based approach without any consideration of wind [17]. Over time, plant growth is affected by wind, according to its strength and duration. Plants respond in three major ways: the girth of the stem is increased, the length of the stem is decreased, and the direction of growth is changed. The leaves of the plant also change in two ways: their thickness is increased and their shape becomes closer to round and stunted $[18,19]$.

This paper proposes the Growth-Flow method for animating the effect of wind on the motion and growth of stems and leaves of virtual plants. Incorporating the effects of wind on growth into a virtual plant that supports motion is an original contribution of this paper. One might assume that plant growth is too slow a process to be considered jointly with plant motion. However, in many uses of virtual plants, the growth is shown at such an accelerated rate that it is reasonable to combine the two. For example, a fast-growing plant may provide a challenge in a computer game, and it will appear more authentic if it also moves with the wind and its growth is affected by the wind. Moreover, a lecturer explaining the effect of wind on long-term growth may want to use an accelerated model to illustrate the interrelationships.

When animating plants, a technique illustrating the combined effect of wind on motion and growth is generally not needed, since only motion is typically of interest. However, when depicting the effect of wind on the growth of plants in an animated scenario, it is likely that one will also want to depict motion. Our experience is that when the wind is acknowledged to have an effect on growth, users want to see evidence of the wind, that is, simultaneous motion due to the 
wind. The Growth. Flow technique described in this paper satisfies this need.

The remainder of this paper is organized as follows. Background information on wind, plant motion, plant growth, and the responses of plants to wind is given in Section 2. Formulae and algorithms for the motion and growth of both stems and leaves in response to wind are given in Section 3. Experimental results are presented in Section 4, a discussion related to current literature is given in Section 5, and conclusions are drawn in Section 6.

\section{Background}

This section describes the motion and growth effects caused by wind. Section 2.1 provides background information concerning wind and the basis of its animation. Section 2.2 provides the background for the effect of wind on plants. Section 2.2.1 describes branch movement, Section 2.2.2 describes branch growth, Section 2.2.3 describes leaf movement, and Section 2.2.4 describes leaf growth.

2.1. Wind Motion. Wind is air in motion. It is produced when atmospheric (air) pressure is higher in one area and lower in another. The velocity of the wind results from the pressure difference between an area of high pressure and an area of low pressure, with higher velocities from greater differences. Wind velocity is characterized by its speed and direction [20]. Existing methods simulate wind by distinguishing between gusts and stable winds $[14,21]$ and by treating it as a fluid flow modeled by the Navier-Stokes equations $[12,22-24]$. We assume that $\mathbf{w}_{t}$, the velocity of the wind at time $t$ everywhere in the simulation, is set according to a wind model. Throughout this paper, all variables shown in bold face represent vectors. Gusts could be modeled in the wind model by rapidly increasing and decreasing the wind speed in particular areas from time to time. Instead, for simplicity, we give leaf movements a random component that can be interpreted by the viewer as wind gusts. This random component is used to decide which of the bending, twisting, and vibrating motions to apply to each leaf.

2.2. Plant Response to Wind. As mentioned in Section 1, plants respond to wind immediately with motion and over time with changes in growth patterns. Plant motion in response to wind has been studied most thoroughly with respect to trees. The response of a tree branch or leaf to wind depends upon its tensile strength, the size of its surface area facing the wind, and its mass $[25,26]$. If a branch has a large surface area facing the wind, a low-tensile strength, and a large mass, it can fracture when the force of the wind exceeds the strength of the branch. Leaves respond in a similar fashion to branches, although with less influence from the leaf's mass, which in negligible, and more from the surface area.

Branch movement in response to wind is observed as a swaying motion, which is also evident in leaf movement. In addition, leaves display a twisting motion in response to the wind and a vibration motion when the wind is tangent to the blade [8].

Plant growth is affected by wind, among many other environmental factors. Several studies have been conducted to discover the response of various plant species to wind. One study observed that elongation was significantly retarded in young plants of Hordeum vulgare, Bryonia dioica, Cucumis sativus, Phaseolus vulgaris, Mimosa pudica, and Ricinus communis [27]. Another observed that leaf growth was inhibited by $30 \%$ in cocklebur plants and grape vines [28]. In addition, when trees of Liquidambar Styraciflua were shaken daily, elongation was reduced by $70 \%-80 \%$ [29]. Recently, a study regarding airflow and plant growth discovered that increased airflow enhanced stem elongation when the plant did not flex its stem and restricted elongation otherwise [30]. Another recent study on wind and mechanical stress on leaves observed that wind restricted growth and also made leaves thicker and closer to being round [18]. As mentioned in Section 1, the overall effect of wind on stems (or branches in trees) is to thicken them, restrict their elongation, and change the direction of their growth and the overall effect on leaves is to thicken them and change their shape closer to being round $[18,19]$.

The remainder of this section describes previous research on plant motion in more detail by considering branch movement, branch growth, leaf movement, and leaf growth.

2.2.1. Branch Movement. Branches are typically modeled using a segment-based approach, exemplified by the work of Sakaguchi and Ohya [1], in which each segment represents an approximately linear part of a branch between nodes. To avoid confusion, in this paper, we reserve the term "node" for its sense in botany, where it refers to a point on a plant where new growth is possible. Typically, a node corresponds to a joint in an animated model. A branch is represented by a computer science tree data structure where the vertices represent segments and the arcs represent the existence of (botany) nodes. The motion of a particular segment depends on the motions of its child segments (i.e., connected segments closer to the external end of the branch) and the effect of the wind on the particular segment. The effect of the wind on a particular segment is calculated based on the combination of wind velocity, axial damping, and restoration force. The axial damping is influenced by the tensile strength of the branch, which depends on its thickness. The restoration force (resistivity) is the force influencing a branch that is not in its rest position to return to that position. Equation (1) can be used to calculate motion of a segment [1]:

$$
\mathbf{N}=\left(\mathbf{F}_{\mathbf{w}}+\mathbf{K}+\mathbf{R}+\mathbf{T}_{\mathbf{r}}\right) \times \mathbf{b},
$$

where $\mathbf{N}$ is the moment of force (torque), $\mathbf{F}_{w}$ is the external force (i.e., wind), $\mathbf{K}$ is the resistivity, $\mathbf{R}$ is the axial damping force at a node, $\mathbf{T}_{r}$ is the force that propagates back from the child branches (if any), $\times$ indicates the cross product, and $\mathbf{b}$ is a vector from the node at the beginning of the segment (where it attaches to its parent segment) to the center of gravity of the segment [1]. The branch movement method described by Sakaguchi and Ohya [1] is illustrated in Figure 1. On the 
TABLE 1: Growth rates for plant species.

\begin{tabular}{|c|c|c|c|}
\hline Species & Branch growth rate & $\begin{array}{l}\text { Change in growth rate due to } \\
\text { wind }\end{array}$ & Reference \\
\hline Aspen (Populus tremula) & $\begin{array}{l}25 \text { or more inches per year (used } \\
\text { Populus tremuloides) }\end{array}$ & $50 \%$ reduction in leaf area & {$[19]$} \\
\hline Bamboo (Poaceaea) & Some grow 39 inches/day & No study found & {$[40]$} \\
\hline Barley & $18 \%$ increase per day & No change in growth rate & {$[41,42]$} \\
\hline Birch (Betula pendula) & $\begin{array}{l}13 \text { or more inches a year (used Betula } \\
\text { papyrifera) }\end{array}$ & $26 \%$ decrease & $\begin{array}{l}\text { [43] (growth rate) } \\
\text { [19] (wind effects) }\end{array}$ \\
\hline Dogwood (Cornus sanguinea) & $45 \mathrm{~cm}$ per year & $20 \%$ reduction in leaf area & $\begin{array}{l}\text { [44] (growth rate) } \\
\text { [19] (wind effects) }\end{array}$ \\
\hline Honeysuckle (Lonicera xylosteum) & $1.5 \mathrm{~m}$ per year & $33.3 \%$ reduction in leaf area & $\begin{array}{l}\text { [45] (growth rate) } \\
{[19] \text { (wind effects) }}\end{array}$ \\
\hline $\begin{array}{l}\text { Lodge Pole Pine (Pinus contortav. } \\
\text { latifolia) }\end{array}$ & 24 or fewer inches per year & 12 to 40 inches per year & [43] (growth rate) \\
\hline $\begin{array}{l}\text { Kidney beans (Phaseolus vulgaris L. cv. } \\
\text { Red Cherokee Wax) }\end{array}$ & $\begin{array}{l}6.4 \mathrm{~mm} / \text { day } \\
\text { ave. increase in elongation per day } \\
0.266 \mathrm{~mm} / \text { day ave. increase in leaf } \\
\text { diameter }\end{array}$ & $\begin{array}{l}1.4 \% \text { decrease in elongation } \\
3.3 \% \text { decrease in leaf diameter }\end{array}$ & {$[46]$} \\
\hline Oak (Quercus robur) & $\begin{array}{l}24 \text { or fewer inches per year (used } \\
\text { White Oak) }\end{array}$ & $25 \%$ reduction in leaf area & $\begin{array}{l}\text { [43] (growth rate) } \\
\text { [19] (wind effects) }\end{array}$ \\
\hline Pea & $11 \%$ increase per day & No change in growth rate & {$[41,42]$} \\
\hline Rape (Brassica napus) & $25 \%$ increase per day & No change in growth rate & {$[41,42]$} \\
\hline White Ash (Fraxinus Americana) & 13 to 24 inches per year & $40 \%$ decrease in leaf area & $\begin{array}{l}\text { [43] (growth rate) } \\
{[19] \text { (wind effects) }}\end{array}$ \\
\hline
\end{tabular}

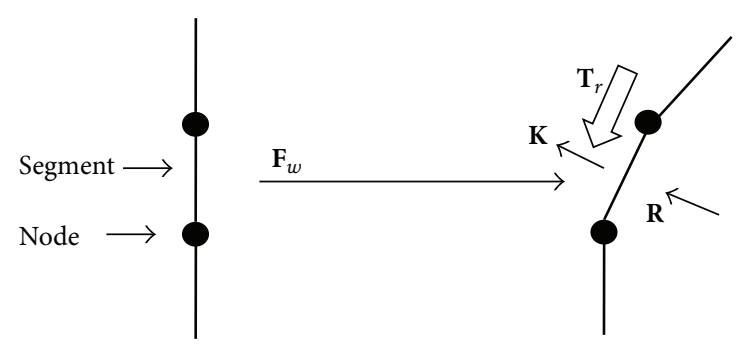

FIGURE 1: Branch movement.

left, three segments joined at two nodes are shown, and on the right, the same segments are shown after the wind has moved the top segments to the right. The segment of interest is the middle one. The forces listed in (1) act in the directions shown.

2.2.2. Branch Growth. Growth is an irreversible increase in size, mass, or number of cells [31]. Ignoring other environmental factors, growth for a living thing, such as a plant, shows no logarithmic, linear, or exponential increase with respect to time, depending on the current time during the year. Three existing ways of measuring growth are (1) absolute growth rate (AGR), which is the total gain in size (height, weight, and radius) or mass (dry or fresh weight) over time, (2) relative growth rate ( $\mathrm{RGR}$ ), which is the change in the logarithm of mass over time, and (3) percentage growth rate (PGR), which is the percentage change in length or mass over time. AGR and RGR are used for measuring growth in plant physiology, whereas PGR is used in horticulture. For illustration AGR, RGR, and PGR can be characterized as shown in (2), (3), and (4), respectively,

$$
\begin{gathered}
\mathrm{AGR}=\frac{\Delta n}{\Delta t}=\frac{n_{2}-n_{1}}{t_{2}-t_{1}}, \\
\mathrm{RGR}=\frac{\Delta n}{\Delta t} \cdot \frac{1}{n}=\frac{\ln n_{2}-\ln n_{1}}{t_{2}-t_{1}}, \\
\mathrm{PGR}=\left(\left(\frac{n_{2} / n_{1}}{t_{2}-t_{1}}\right) * 100 \%\right)-100 \%,
\end{gathered}
$$

where $n_{1}$ and $n_{2}$ are the relevant size or mass measurements and $t_{1}$ and $t_{2}$, where $t_{2}>t_{1}$, are the respective times at which these measurements were taken.

Branch growth is seasonal. When it occurs, a sigmoidal growth curve is observed such that the growth rate slowly increases during the early part of the growing season, remains constant (linear growth) during the main part of the growing season, and then declines so that it reaches zero when the segment achieves its maximum size [31]. Stem elongation proceeds differently among plants: some grow at the apical meristem (the branch tip) resulting in long older growth, some exhibit varying rates of elongation over several segments, and some grow evenly along all stem segments. For simplicity, we assume the later. Given this assumption, the principal differentiating characteristic is the growth rate during the linear-growth period. Absolute and percentage growth rates for various plants are provided in Table 1 in the exact form given in the listed references, along with wind inhibition percentages, which will be discussed shortly. 


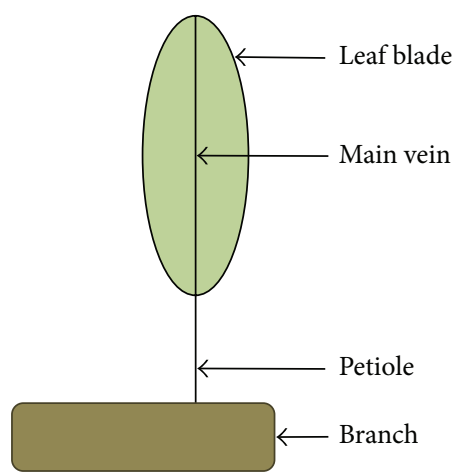

FIGURE 2: Leaf with petiole connected to a branch.

We are presenting a scattering of examples because apparently no large-scale-comparative study has been reported.

Branch growth is affected by wind through a thickening of the stem [32-36] and reduced elongation [37-39]. The direction of the branch is altered to align more closely with the wind vector if the wind is constant and strong over the lifetime of the plant. In general, the effect of wind on growth is not significant unless the wind persists over long periods and has sufficient speed, for example, over 4 meters per second for aspen [19]. The amount of effect on growth is correlated with wind speed [19]. Table 1 also includes the observed effect of wind on growth rate for the sample plants where available.

Another common growth formula is based on the Richards growth model [47-49] which considered exponential growth as described in

$$
y_{t}=A\left(1+b e^{-k t}\right)^{1 / 1-m}, \quad m>1, b>1, k>0, t \geq 0,
$$

where $t$ is time, $y_{t}$ is the size of the growing part (e.g., amount of dry matter) at time $t, A$ is the asymptotic value of the size, and $m, b$, and $k$ are constant coefficients, where $m$ determines the curve type, $b$ describes the linear component of growth, and $k$ determines the rate at which growth changes over time [47]. According to Richards, " $k$ is the "rate constant" which determines the spread of the curve along the time axis, $b$ is usually unimportant biologically, since it reflects only the choice of the zero of the time" ([47], p. 290). (This $b$ should not to be confused with the vector $\mathbf{b}$ used elsewhere in this paper.) The constant $m$ influences the shape of the curve. The formula used within the Growth-Flow method resembles this growth formula.

2.2.3. Leaf Movement. A leaf is attached to a petiole, which is in turn attached to a branch, as illustrated in Figure 2. A petiole is a smaller branch structure that ensures proper attachment of the leaf to the branch. The movement of the petiole influences the movement of the leaf and vice versa. The tensile strength of the petiole influences how far a leaf bends and whether the petiole breaks off its branch. The surface area of the leaf blade is also a contributing factor to the motion of the leaf in response to wind, with a larger surface area resulting in a stronger response. The tensile strength of the blade, which depends on the strength of the vein, also influences the movement of the leaf. If a leaf has been exposed to drought and its tensile strength is low, its blade can bend more in response to the wind than it would otherwise. All the previously discussed factors, that is, wind speed, tensile strength of petiole, tensile strength of blade, and leaf blade surface area, contribute to the overall leaf motion. The first three of these factors are considered in more details in Section 3.

2.2.4. Leaf Growth. Steady wind of sufficient speed has been observed to affect the growth of a leaf through an increase in the thickness of the leaf blade. For aspen, this effect was observed for wind with a speed of more than 4 meters per second [19]. In addition, leaves tend to become smaller in surface area [19] and closer to being round [18] due to wind stresses on their surfaces.

\section{The Growth-Flow Method}

The Growth-Flow method has two components resulting from wind influence: physical motion and growth effects on branches and leaves. The methods used to simulate the physical motion and growth for a branch are described in Section 3.1, and those for a leaf are described in Section 3.2.

3.1. Branch Response to Wind. To simulate the physical motion and growth for a branch, we consider the movement in Section 3.1.1 and the growth in Section 3.1.2.

3.1.1. Branch Movement. Branch movement in response to wind was modeled by swaying the branch toward the wind vector and back over time. To explain in a simple fashion, consider a 2D example, as illustrated in Figure 3, which uses a right-handed coordinate system: part (a) shows the centers of rotation of a plant (which correspond to the nodes); part (b) shows that as the wind pushes left on a branch segment, the angle of rotation increases by $\theta$ around the center of rotation for that branch segment, and part (c) shows that the angle of rotation is decreased as the branch returns to its original position due to the restorative force and tensile strength of the branch. To produce the rotation, the value for $\theta$ is increased over half a time period until a certain angle is reached and then it is decreased back to its original value over the other half of the time period. To achieve a natural slowing down and speeding up at the ends of the arcs, a sinusoidal ease-in/easeout function is used [50].

Although the example was shown in $2 \mathrm{D}$, the wind vector is actually $3 \mathrm{D}$. Given the branch direction $\mathbf{b} / b$ and wind direction $\mathbf{w} / w$ defined in the same coordinate system, the axis of rotation for the wind effect is obtained by taking their cross product $\mathbf{b} / b \times \mathbf{w} / w$. The amount to rotate around this axis is determined based on the angular acceleration, calculated by dividing the torque from (1) by the mass of the branch. The maximum rotation, which would cause the branch to align with the wind vector, is $\cos ^{-1}(\mathbf{b} \cdot \mathbf{w} / b w)$. The mass of a branch is arbitrarily set to 10 times the mass of a leaf. A leaf is thus affected 10 times more than a branch by the wind.

3.1.2. Branch Growth. To represent the effect of wind on growth, we assume that there is a species-specific minimum 


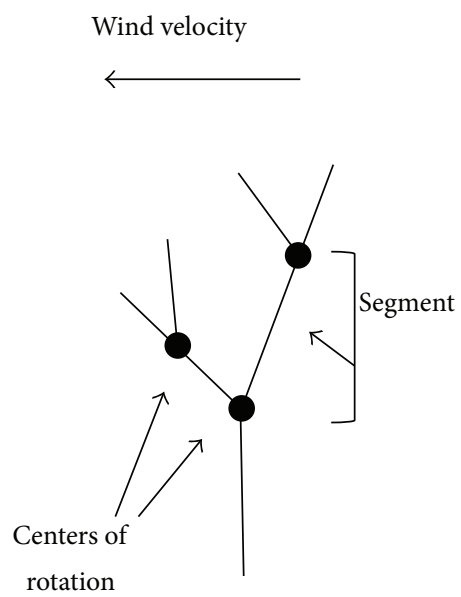

(a) Original branch position
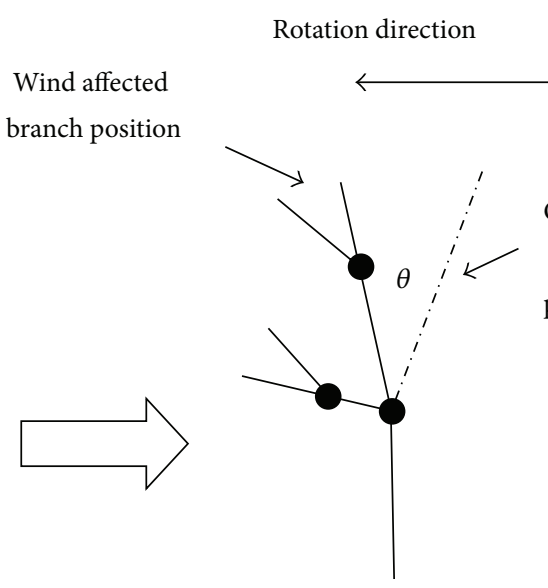

(b) Branch moving left
Rotation direction

Original

branch

position
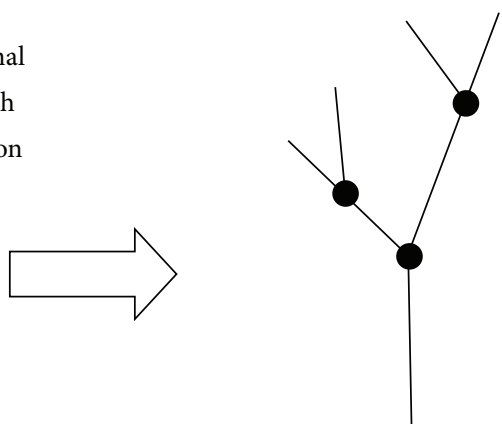

(c) Branch moving right

Figure 3: Branch segments and nodes.

Input: time $t$, branch vector $\mathbf{b}$, and wind velocity $\mathbf{w}$.

Output: branch girth $r_{t}$, branch length $l_{t}$, and branch growth angle $\alpha_{t}$, all at time $t$.

Set the wind growth factor (see (6)).

Set the branch radius (see (7)).

Set the branch length (see (8)).

If $l_{t} \geq l_{\max }$, then $l_{t}=l_{\max }$.

Set the growth angle (see (9)).

Determine the axis of rotation for the branch as $\mathbf{u}=\mathbf{b} \times \mathbf{w}$.

Rotate the branch around axis $\mathbf{u}$ according to the growth angle $\alpha_{t}$.

Additionally rotate the branch around axis $\mathbf{u}$ according to wind velocity $\mathbf{w}$ (1).

Algorithm 1: The general algorithm for branch growth and movement.

speed $w_{\min }$, and maximum speed $w_{\max }$, such that growth is not affected if the wind is less than or equal to $w_{\min }$ and that the full effect occurs if the wind is greater than or equal to $w_{\max }$. We assume that the effect is linearly interpolated between these speeds. To quantify the effect of wind on growth, we define the wind growth factor $f(w)$, which yields a value in the range $[0,1]$, as follows:

$$
f(w)=\min \left(1, \max \left(0, \frac{w-w_{\min }}{w_{\max }-w_{\min }}\right)\right) .
$$

The three effects on branch growth are simulated: girth increase, elongation reduction, and cumulative change in growth angle (henceforth called the growth angle). For a wind with uniform velocity $\mathbf{w}$ (and constant speed $w$ ), we use (7), (8), and (9) for these three factors:

$$
\begin{gathered}
r_{t}=r_{0}\left(1+g_{r} \Delta r\right)^{t} \quad \text { where } \Delta r=1+s_{r} f(w), \\
l_{t}=l_{0}\left(g_{l} \Delta l\right)^{t}, \quad \text { where } \Delta l=1-s_{l} f(w), \\
\alpha_{t}=\alpha_{0}(1+\Delta \alpha)^{t}, \quad \text { where } \Delta \alpha=c_{\alpha} s_{\alpha} f(w),
\end{gathered}
$$

where $t$ is the elapsed time; $r_{t}, l_{t}$, and $\alpha_{t}$ are the radius (girth), length, and growth angle at time $t ; r_{o}, l_{o}$, and $\alpha_{o}$ are the original radius, length, and growth angle at time $0 ; g_{r}$ and $g_{l}$ are the normal rates of radius growth and branch elongation; $\Delta r, \Delta l$, and $\Delta \alpha$ are the proportional changes in radius growth, branch elongation and growth angle due to the wind, respectively; and $c_{\alpha}$ is a conversion constant from meters per second to degrees per second. The species-specific constants $s_{r}, s_{l}$, and $s_{\alpha}$, are assumed to be in the range $[0,1]$. Equations $(7)$ and (8) specify that the changes are affected by both the speed and the duration of the wind but the effect of the wind direction is negligible. If the length reaches a maximum determined by the species, no further increase in length occurs. If the wind speed is less than a species-specific minimum speed $w_{\text {min }}$, then growth is not affected by the wind.

\subsubsection{Branch Growth and Motion. See Algorithm 1.}

3.2. Leaf Response to Wind. The leaf response to the wind is modeled by considering movement and growth, as discussed in Sections 3.2.1 and 3.2.2, respectively. 


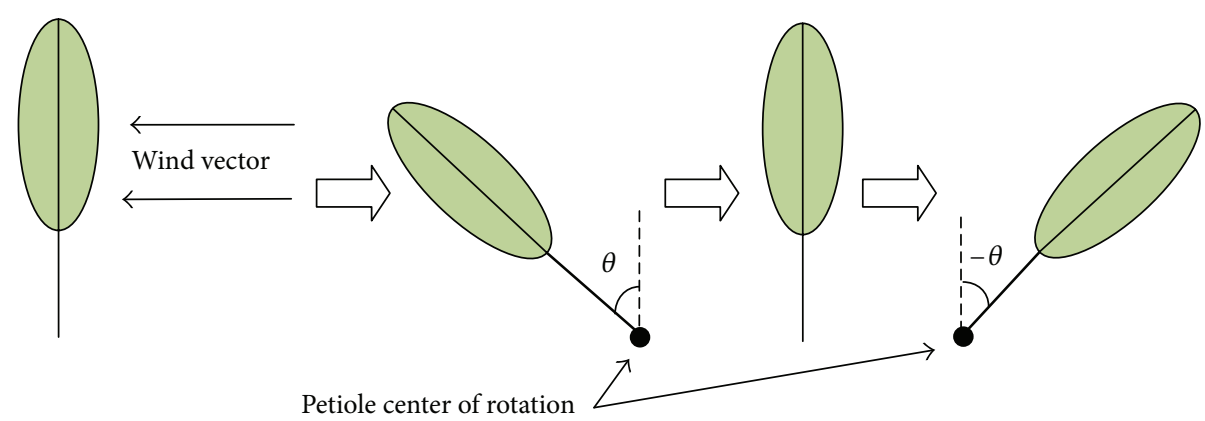

FIGURE 4: Petiole bending in response to wind.

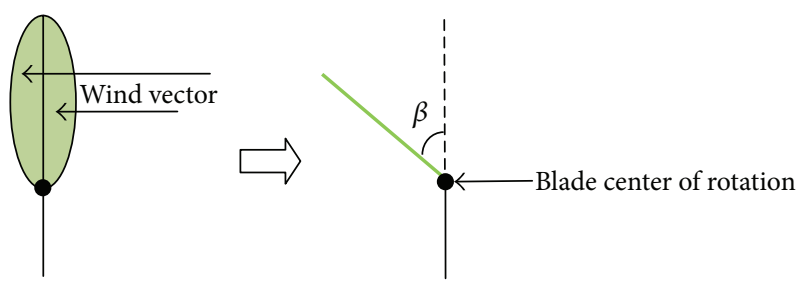

FIGURE 5: Blade bending in response to wind.

3.2.1. Leaf Movement. The leaf blade moves in response to wind in one of three ways: bending, twisting, and vibrating. Bending is the motion when the leaf blade is facing the wind (perpendicular to the wind). Twisting is the motion where the leaf blade turns in response to the wind, which occurs when the blade is not perpendicular to the wind. Vibration is tiny movements along the petiole/leaf axis when the leaf blade is parallel to the wind. The petiole response to wind is limited to bending, because vibration and twisting are negligible due to the small surface area. Another consideration is the effect of neighbouring leaves on the motion of the leaf where there is collision and bounce. For the Growth-Flow approach, we ignore these effects based on our assumption that they are relatively small.

As mentioned in Section 2, to model the effects of gusts of wind, the Growth-Flow approach includes a stochastic parameter that influences leaf motion. Let $l_{s} \in[0,1]$ represent the fraction of the wind's velocity that affects a leaf at the current time. This factor is incorporated in the equations for leaf motion. The remainder of this subsection describes the Growth-Flow approach to leaf motion by considering each of bending, twisting, and vibrating. Throughout, we use time $\Delta t$ to represent the time interval, $t_{p}$ to represent the tensile strength of the petiole, and $t_{b}$ to represent the tensile strength of the blade.

(1) Bending. In the Growth-Flow approach, if a leaf blade is facing (perpendicular to) the wind, then bending occurs either at the petiole center of rotation, as illustrated in Figure 4, or at the blade center of rotation, as illustrated in Figure 4. If the tensile strength of the petiole is less than that of the blade, that is, $t_{p} \leq t_{b}$, then any bending will be petiole bending; otherwise it will be blade bending. We denote the amount of petiole bending by $\theta$ and the amount of blade bending by $\beta$. Blade bending occurs if the tensile strength of the blade is less than the force of the wind, as illustrated in Figure 4 . In either case, we model the bending as a rotation at a single pointas follows:

$$
\begin{gathered}
s_{t}= \begin{cases}-s_{t-1}, & \text { if } \theta_{t-1}+s_{t-1} w c_{\theta} \Delta t>\theta_{\max } \\
-s_{t-1}, & \text { if } \theta_{t-1}+s_{t-1} w c_{\theta} \Delta t<\theta_{\min } \\
s_{t-1}, & \text { otherwise }\end{cases} \\
\theta_{t}=\theta_{t-1}+s_{t} w c_{\theta} \Delta t
\end{gathered}
$$

The direction of bending, $s_{t} \in\{1,-1\}$, stays constant until the sum of the previous angle of rotation, $\theta_{t-1}$, plus an update of $w c_{\theta} \Delta t$ in the same direction would be higher than the maximum angle $\theta_{\max }$ or lower than the minimum angle $\theta_{\min }$, in which case $s_{t}$ switches, as described in (10). The update to the bending, $s_{t} w c_{\theta} \Delta t$, is computed based on the sign, $s_{t}$, the wind speed, $w$, a conversion constant, $c_{\theta}$, over a change in time $\Delta t$. The complete motion, shown in Figures 5 and 6 , is accomplished by incrementing the current angle with the change in angle at the center of rotation, as described by (11). If the leaf is oriented on the $X Y$ plane, bending occurs in the $Z$ dimension.

(2) Twisting. Another motion leaves exhibit is twisting around the petiole. We modeled twisting as a rotation of the petiole at the point where it joins the branch. We assume that in the local frame of reference the leaf is initially lying on the $X Y$ plane and the branch is aligned roughly with the $Y$ axis. The amount of twisting, $\psi$, is incremented/decremented as specified by the sign, $s_{t}$, over a change in time, $\Delta t$, influenced by the wind speed, $w$, subject to a conversion constant $c_{\psi}$, in the range from $\psi_{\min }$ to $\psi_{\max }$, as described in (12). The complete motion, which is shown in Figure 7, is accomplished by incrementing the current angle with the change in angle, as described by

$$
\begin{aligned}
& s_{t}= \begin{cases}-s_{t-1}, & \text { if } \psi_{t-1}+s_{t-1} w c_{\psi} \Delta t>\psi_{\max } \\
-s_{t-1}, & \text { if } \psi_{t-1}+s_{t-1} w c_{\psi} \Delta t<\psi_{\min } \\
s_{t-1}, & \text { otherwise }\end{cases} \\
& \psi_{t}=\psi_{t-1}+s_{t} w c_{\psi} \Delta t .
\end{aligned}
$$

(3) Vibrating. The third motion a leaf exhibits is vibration, as illustrated in Figure 8. If the leaf is oriented flat on the 


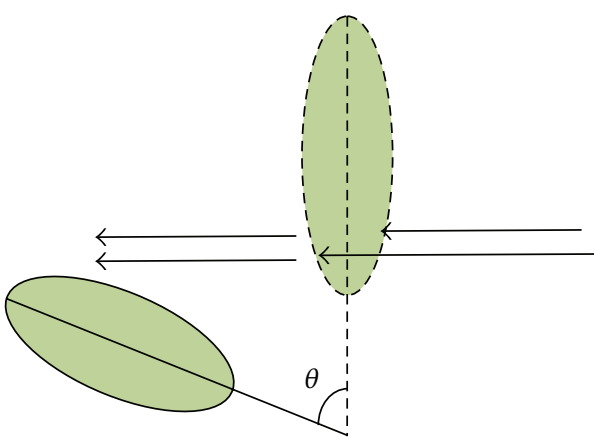

(a)

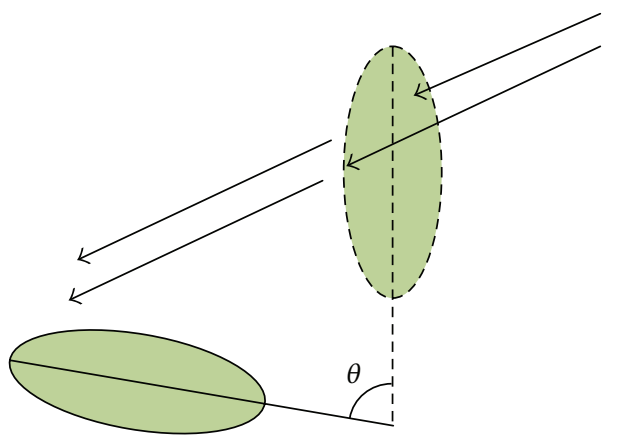

(c)

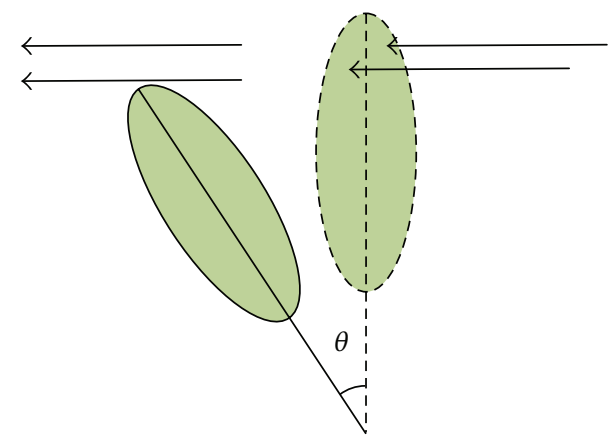

(b)

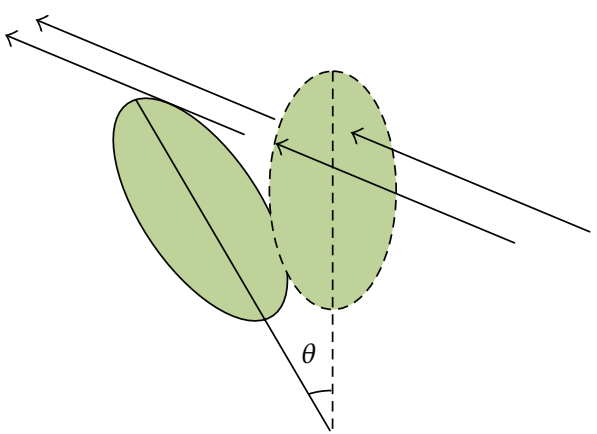

(d)

FIGURE 6: Various responses to wind duration and velocity on leaf blade: (a) wind is perpendicular to blade and hits its lower half, (b) wind is perpendicular to blade and hits its upper half, (c) wind is oblique to blade and hits its upper half, and (d) wind is oblique to blade and hits its upper half.

$X Y$ plane, vibration is in the $Z$ dimension. The amount of vibration, $\varphi$, is incremented/decremented as specified by the sign, $s_{t}$, over a change in time, $\Delta t$, influenced by the wind speed, $w$, subject to a conversion constant $c_{\varphi}$, in the range from $\varphi_{\min }$ to $\varphi_{\max }$, as described in (14). The complete motion, which is shown in Figure 8, is accomplished by incrementing the current angle with the change in angle, as described by (15)

$$
\begin{aligned}
s_{t}= \begin{cases}-s_{t-1}, & \text { if } \varphi_{t-1}+s_{t-1} w c_{\varphi} \Delta t>\varphi_{\max } \\
-s_{t-1}, & \text { if } \varphi_{t-1}+s_{t-1} w c_{\varphi} \Delta t<\varphi_{\min } \\
s_{t-1}, & \text { otherwise }\end{cases} \\
\varphi_{t}=\varphi_{t-1}+s_{t} w c_{\varphi} \Delta t .
\end{aligned}
$$

The three equations are the same except for varying angles of rotation and varying values for the maximum and minimum angles of rotation. They can be implemented with a single function with different values for its parameters.

3.2.2. Leaf Growth. Changes in leaf thickness and leaf radius are modeled in (16) and (17), respectively, as follows:

$$
\begin{gathered}
h_{t}=h_{0}\left(1+g_{h} w s_{h}\right)^{t}, \\
d_{t}=d_{0}\left(g_{d} w s_{d}\right)^{t},
\end{gathered}
$$

where $t$ is the elapsed time, $h_{t}$ is the resulting leaf thickness at time $t, h_{0}$ is the original leaf thickness, $g_{h}$ is the normal leaf thickness growth rate, $s_{h}$ is a species specific constant, $d_{t}$ is the resulting leaf radius at time $t, d_{0}$ is the original leaf radius, $g_{d}$ is the normal leaf growth rate, $\Delta d$ is the proportional change in the radius, and $s_{d}$ is a species specific constant.

\subsubsection{Leaf Growth and Motion. See Algorithm 2.}

\section{Results}

The tree branch, petiole, and vein structure were generated using a nested L-System [51], and the leaves were generated using the Radius Variation method [52]. Figure 11 demonstrates the effect of a $10 \mathrm{~km} / \mathrm{h}$, left-to-right wind on a tiny branch with four leaves attached by petioles over one full cycle of swaying motion. To simplify viewing, in this figure, the branch is shown at one-third scale and the leaves and petioles have their normal size. During the first 16 frames, the branch is moving from right to left. For example, the leftmost petiole visible in the first frame can be seen (where visible) to gradually move to the left in the first 16 frames. Then it moves back to the center over the next 16 frames, it continues to the right over 16 more frames, and finally back to the left over the remaining 16 frames to complete the cycle. The leaves also exhibit a variety of bending, twisting, and vibrating behaviors. For each frame, one of the three behaviors is randomly selected for updating ( $1 / 3$ probability each). We set the maximum and minimum angles for these behaviors as $\theta_{\min }=-5, \theta_{\max }=5, \beta_{\min }=-10, \beta_{\max }=10$, 
Global Initialize: $t=0, s_{0}=1, \theta_{0}=0, h_{0}=1, d_{0}=1$

Choose a random type of leaf motion $r \in\{1,2,3\}$

Input: Time $t$, old leaf parameters $\left(\theta_{t-1}, \beta_{t-1}, \psi_{t-1}, \varphi_{t-1}\right)$, wind speed $w$

Output: New leaf parameters $\left(\theta_{t}, \beta_{t}, \psi_{t}, \varphi_{t}\right)$, thickness $\left(h_{t}\right)$, and radius $\left(d_{t}\right)$

If $w>c_{p} t_{p}$ or $w>c_{b} t_{b}$, that is, if the wind exceeds the maximum tensile strengths of the petiole $\left(t_{p}\right)$ or blade $\left(t_{b}\right)$, then exit because the leaf is no longer attached.

$\theta_{t}=\theta_{t-1}, \beta_{t}=\beta_{t-1}, \psi_{t}=\psi_{t-1}$, and $\varphi_{t}=\varphi_{t-1}$

If $r=1$

If $t_{p} \leq t_{b}$

Bend the petiole at the petiole center of rotation

else

(See (10) and (11))

Bend the leaf blade at the leaf center of rotation

else if $r=2$

$\beta_{t}=\beta_{t-1}+s_{t} w c_{\beta} \Delta t((11)$ with $\beta$ instead of $\theta)$

Twist the leaf (see (12) and (13))

else if $r=3$

Vibrate the leaf at the leaf center of rotation (see (14) and (15))

Calculate the leaf thickness (see (16))

Calculate the leaf radius (see (17))

Algorithm 2: The general algorithm for growth and movement of one leaf.

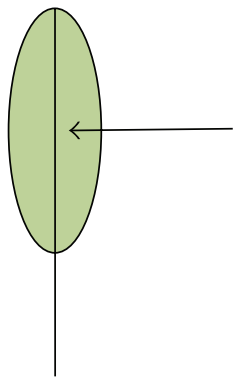

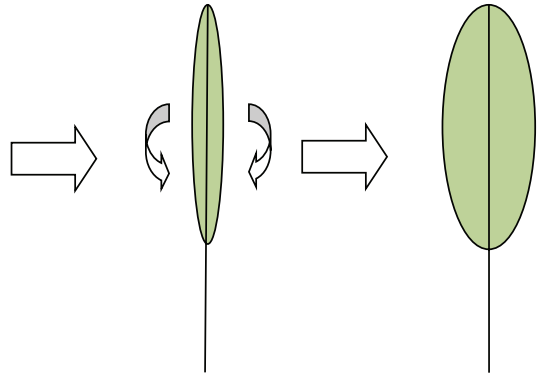

(a) Side view
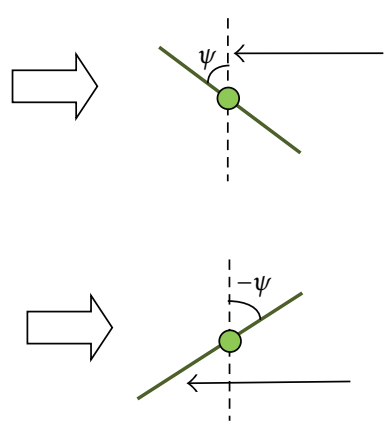

(b) Top view

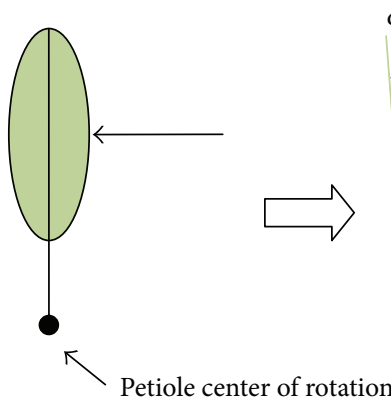

Figure 8: Leaf vibration in response to wind.

Further illustration of the response to wind is provided in Figure 10 for a seven-year-old tree. As in Figure 9, the tree trunk and branches are shown at a smaller scale.

The Growth-Flow method, in addition to supporting full motion response of branches and leaves to the wind, also limits growth as illustrated in Figure 11. Parts (a) and (b) of this figure demonstrate growth over a two-second sample without wind and with wind, respectively. There is a marked difference in the plant's growth with the branch length and leaf size both reduced when wind is present, which matches observations about natural trees.

As shown by the above results, the Growth-Flow method is effective at calculating leaf behavior in response to wind, both with respect to motion (Figures 9 and 10) and growth (Figure 11). The wind effect on plant motion results in the bending, swaying, and twisting of the plant. The wind effect on plant growth results in thickening of leaf blade, reduction of leaf radius, reduction in stem elongation, and increase in stem radius and influences the direction of growth to $\psi_{\min }=-15, \psi_{\max }=15, \varphi_{\min }=-5$, and $\varphi_{\max }=5$. Experiments with other ranges for the three behaviors also resulted in apparently natural plant behavior, although the ranges chosen seemed the most realistic visually. Overall, as Figure 9 illustrates, the responses of the leaves and the branches to wind do mimic nature. 


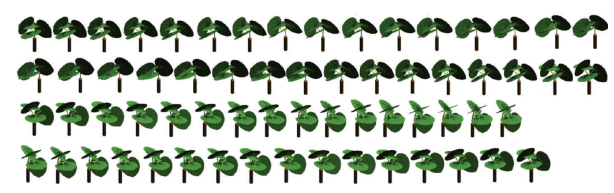

Figure 9: Response of petiole and leaves to wind: consecutive frames of animation.

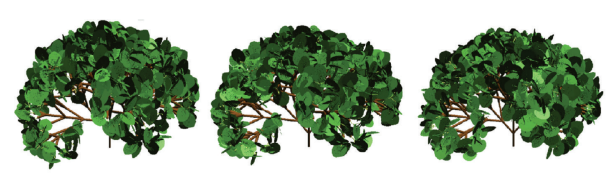

FIgURE 10: Year Seven motion in response to wind, images taken four seconds apart.

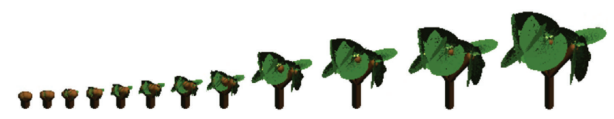

(a) With no wind

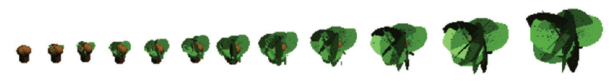

(b) With wind

FIGURE 11: Growth for two seconds with and without wind.

align more closely with the wind vector, when its direction is consistent for a length of time.

\section{Discussion}

The Growth-Flow method for animating plants while considering the influence of both motion and growth, can be contrasted with previous work. Crucially, none of the previous work considers the effect of growth on plant animation. In particular, the most similar approaches to plant motion to the one adopted here, including the segment-based [1], stochastic-based [6], and physics-based [1-3, 24] methods, did not consider growth factors. Feng et al. [14] proposed a method based on only two rotations local to the leaf instead of the three rotations we consider. Although growth is not considered, Ota et al. [53] use $1 / f \beta$ noise functions with three movements (horizontal, vertical, and rotational) to provide a more sophisticated stochastic model of plant motions. Our method focuses on the local behavior of the leaf in response to wind. In contrast, the method proposed by Zioma [8] considers the lifting effects of wind on groups of leaves, but does not consider the response of individual leaves. Akagi and Kitajima [2] devised a boundary condition map to locate tree parts in a simplified grid, which reduces the number of calculations needed for motion simulation, but ignored the effect of wind on growth.

The current paper considers branch elongation, branch radius increase, leaf thickening, leaf radius decrease, and change in branch growth angle. Other diverse approaches have been devised for animating plant motion, including simulating motion with filtered uncorrelated noise in a frequency domain $[7,53,54]$, simulating overall motion by using rotations based on torque and oscillation $[55,56]$, using massspring networks $[57,58]$, displaying texels with billboarding techniques [59], and using a finite element model (FEM) [60$62]$. None of these diverse methods included growth.

\section{Conclusion}

Both motion and growth of branches and leaves are influenced by the wind. The Growth-Flow method proposed in this paper is the only method known for plant animation that considers both. This method, as illustrated in Figures 9 to 11, includes the following changes to growth: decrease in branch elongation, increase in branch radius, change in branch growth angle, increase in leaf thickness, and decrease in leaf size. All of these effects were simulated and match the observed response of plants to wind duration and velocity over extended periods of time.

Further study is required to detect collisions between plant parts and to add the influence of petiole and leaf motions to those of the branches. In addition utilization of a global approach to incorporate leaf-petiole-branch interactions requires investigation. Growth is also influenced by other environmental factors, such as temperature and nutrient, carbon dioxide, and water availability, which could be incorporated in to the algorithm as well.

\section{References}

[1] T. Sakaguchi and J. Ohya, "Modeling and animation of botanical trees for interactive virtual environments," in Proceedings of the ACM Symposium on Virtual Reality Software and Technology (VRST '99), pp. 139-146, December 1999.

[2] Y. Akagi and K. Kitajima, "Computer animation of swaying trees based on physical simulation," Computers and Graphics, vol. 30, no. 4, pp. 529-539, 2006.

[3] R. Habel, A. Kusternig, and M. Wimmer, "Physically guided animation of trees," Computer Graphics Forum, vol. 28, no. 2, pp. 523-532, 2009.

[4] L. Ding, C. Chongcheng, T. Liyu, and W. Qinmin, "Geometrical shapes and swaying movements of realistic tree: design and implementation," in Proceedings of the 8th International Conference on Virtual Reality Continuum and Its Applications in Industry (VRCAI '09), pp. 295-302, ACM, New York, NY, USA, December 2009.

[5] H. Ono, "Practical experience in the physical animation and destruction of trees," in Proceedings of the Eurographics Workshop on Animation and Simulation, D. Thalman and M. van de Panne, Eds., pp. 149-159, Springer, New York, NY, USA, 1997.

[6] M. Shinya and A. Fournier, "Stochastic motion-motion under the influence of wind," Computer Graphics Forum, vol. 11, no. 3, pp. 119-128, 1992.

[7] S. Ota, M. Tamura, T. Fujimoto, K. Muraoka, and N. Chiba, "A hybrid method for real-time animation of trees swaying in wind fields," Visual Computer, vol. 20, no. 10, pp. 613-623, 2004.

[8] R. Zioma, "GPU-generated procedural wind animations for trees," in GPU Gems 3, Addison-Wesley Professional, 2007.

[9] J. Long, C. Reimschussel, O. Britton, and M. Jones, "Motion capture for natural tree animation," in SIGGRAPH 2009: Talks 
(SIGGRAPH '09), New Orleans, La, USA, August 2009, article 77.

[10] J. Beaudoin and J. Keyser, "Simulation levels of detail for plant motion," in Proceedings of the ACM SIGGRAPH/Eurographics Symposium on Computer Animation, pp. 297-304, 2004.

[11] M. Yang, B. Sheng, E. Wu, and H. Sun, "Multi-resolution tree motion synthesis in angular shell space," in Proceedings of the 8th International Conference on Virtual Reality Continuum and Its Applications in Industry (VRCAI '09), pp. 47-52, December 2009.

[12] J. Wejchert and D. Haumann, "Animation aerodynamics," Computer Graphics, vol. 25, no. 4, pp. 19-22, 1991.

[13] C. Jurasek, P. Prusinkiewicz, and B. Moula, "Integrating biomechanics into developmental plant models expressed using Lsystems," in Plant Biomechanics, H. Ch. Spatz and T. Speck, Eds., pp. 615-624, Badenweiler, Freiburg, Germany, 2000.

[14] J. Feng, Y. Chen, T. Yan, and E. Wu, "Leaf movement simulation," Journal of Computer Science and Technology, vol. 16, no. 2, pp. 189-192, 2001.

[15] S. Lu, X. Guo, C. Zhao, and C. Li, "Model and animate plant leaf wilting," in Technologies for E-Learning and Digital Entertainment, Z. Pan, X. Zhang, A. El Rhalibi, W. Woo, and Y. Li, Eds., vol. 5093 of Lecture Notes in Computer Science, pp. 728-735, Springer, Berlin, Germany, 2008.

[16] G. Wu, L. WenHui, and F. Guanghui, "Physically based animation of broad-leaf plant," International Journal of Computer Science and Network Security A, vol. 6, no. 2, pp. 198-204, 2006.

[17] I. R. Wang, J. W. L. Wan, and G. V. G. Baranoski, "Physicallybased simulation of plant leaf growth," Computer Animation and Virtual Worlds, vol. 15, no. 3-4, pp. 237-244, 2004.

[18] N. P. R. Anten, R. Alcalá-Herrera, F. Schieving, and Y. Onoda, "Wind and mechanical stimuli differentially affect leaf traits in Plantago major," New Phytologist, vol. 188, no. 2, pp. 554-564, 2010.

[19] W. Flückiger, J. J. Oertli, and H. Flückiger-Keller, "The effect of wind gusts on leaf growth and foliar water relations of aspen," Oecologia, vol. 34, no. 1, pp. 101-106, 1978.

[20] E. Boeker and R. van Grondelle, Environmental Physics: Sustainable Energy and Climate Change, John Wiley and Sons, 3rd edition, 2011.

[21] X. Ao, Z. Wu, and M. Zhou, "Real time animation of trees based on BBSC in computer games," International Journal of Computer Games Technology, vol. 2009, Article ID 970617, 8 pages, 2009.

[22] J. Stam, "Stable fluids," in Proceedings of the 26th Annual Conference on Computer Graphics and Interactive Techniques (SIGGRAPH '99), pp. 121-128, 1999.

[23] F. Perbet and M. P. Cani, "Animating prairies in real-time," in Proceedings of the Symposium on Interactive 3D Graphics, pp. 103-110, March 2001.

[24] N. J. Oliapuram and S. Kumar, "Realtime forest animation in wind," in Proceedings of the 7th Indian Conference on Computer Vision, Graphics and Image Processing (ICVGIP '10), pp. 197204, ACM, December 2010.

[25] K. R. James, N. Haritos, and P. K. Ades, "Mechanical stability of trees under dynamic loads," American Journal of Botany, vol. 93, no. 10, pp. 1522-1530, 2006.

[26] S. Vogel, "Drag and reconfiguration of broad leaves in high winds," Journal of Experimental Botany, vol. 40, no. 8, pp. 941948, 1989.

[27] M. J. Jaffe, “Thigmomorphogenesis: the response of plant growth and development to mechanical stimulation-with special reference to Bryonia dioica," Planta, vol. 114, no. 2, pp. 143-157, 1973.

[28] F. B. Salisbury, The Flowering Process, Macmillan, New York, NY, USA, 1963.

[29] P. L. Neel and R. W. Harris, "Motion-induced inhibition of elongation and induction of dormancy in liquidambar," Science, vol. 173, no. 3991, pp. 58-59, 1971.

[30] V. C. Smith and A. R. Ennos, "The effects of air flow and stem flexure on the mechanical and hydraulic properties of the stems of sunflowers Helianthus annuus L," Journal of Experimental Botany, vol. 54, no. 383, pp. 845-849, 2003.

[31] P. F. Wareing and I. D. J. Phillips, Growth and Differentiation in Plants, Pergamon Press, Oxford, UK, 1981.

[32] M. R. Jacobs, "The effect of wind sway on the form and development of Pinus radiate D. Don.," Australian Journal of Botany, vol. 2, no. 1, pp. 35-51, 1954.

[33] F. W. Telewski and M. J. Jaffe, "Thigmomorphogenesis: field and laboratory studies of Abies fraseri in response to wind or mechanical perturbation," Physiologia Plantarum, vol. 66, pp. 211-218, 1986.

[34] A. Stokes, A. H. Fitter, and M. P. Coutts, "Responses of young trees to wind and shading: effects on root architecture," Journal of Experimental Botany, vol. 46, no. 290, pp. 1139-1146, 1995.

[35] C. Mattheck, Trees: The Mechanical Design, Springer, Berlin, Germany, 1991.

[36] A. M. Goodman and A. R. Ennos, "A comparative study of the response of the roots and shoots of sunflower and maize to mechanical stimulation," Journal of Experimental Botany, vol. 47, no. 303, pp. 1499-1507, 1996.

[37] F. H. Whitehead, "Experimental studies on the effect of wind on plant growth and anatomy," New Phytologist, vol. 61, no. 1, pp. 59-62, 1962.

[38] R. O. Lawton, "Wind stress and elfin stature in a montane rain forest tree: an adaptive explanation," American Journal of Botany, vol. 69, no. 8, pp. 1224-1230, 1982.

[39] C. Coutand and B. Moulia, "Biomechanical study of the effect of a controlled bending on tomato stem elongation: local strain sensing and spatial integration of the signal," Journal of Experimental Botany, vol. 51, no. 352, pp. 1825-1842, 2000.

[40] D. Farrelly, The Book of Bamboo, Sierra Club Books, 1984.

[41] R. M. Wadsworth, "An optimum wind speed for plant growth," Annals of Botany, vol. 23, no. 1, pp. 195-199, 1959.

[42] R. M. Wadsworth, "The effect of artificial wind on the growthrate of plants in water culture: with plate and three figures in the text," Annals of Botany, vol. 24, no. 2, pp. 200-211, 1960.

[43] M. A. Dirr, Manual of Woody Landscape Plants. Their Identification, Ornamental Characteristics, Culture, Propagation and Uses, Stipes Publishing L.L.C, Champaign, Ill, USA, 5th edition, 1998.

[44] Royal Horticultural Society, 2011, http://www.hedgenursery.co .uk/product/common-dogwood-cornus-sanguinea-80-100 cmbare-root-2yr-11-pack-of-25-H80CORS11.html.

[45] A. D. Leatherman, Ecological life-history of Lonica japonica Thunb [Ph.D. thesis], University of Tennessee, 1955.

[46] E. R. Hunt Jr. and M. J. Jaffe, "Thigmomorphogenesis: the interaction of wind and temperature in the field on the growth of Phaseolus vulgaris L.," Annals of Botany, vol. 45, no. 6, pp. 665-672, 1980.

[47] F. J. Richards, "A flexible growth function for empirical use," Journal of Experimental Botany, vol. 10, no. 2, pp. 290-301, 1959. 
[48] F. J. Richards, "The quantitative analysis of growth," in Plant Physiology: A Treatise, F. C. Steward, Ed., pp. 3-76, Academic Press, New York, NY, USA, 1969.

[49] A. Gregorczyk, "Richards plant growth model," Journal of Agronomy and Crop Science, vol. 181, no. 4, pp. 243-247, 1998.

[50] R. Parent, Computer Animation: Algorithms and Techniques, Morgan Kaufmann, 3rd edition, 2010.

[51] P. Prusinkiewicz and A. Lindenmayer, The Algorithmic Beauty of Plants, Springer, 1990.

[52] T. L. M. Derzaph and H. J. Hamilton, "Parametric methods for generating leaf geometry," Tech. Rep. CS-2013-02, Department of Computer Science, University of Regina, Regina, Canada, 2013.

[53] S. Ota, T. Fujimoto, M. Tamura, K. Muraoka, K. Fujita, and N. Chiba, " $1 / f \beta$ noise-based real-time animation of trees swaying in wind fields," in Proceedings of the Computer Graphics International, pp. 52-59, July 2003.

[54] J. Stam, "Stochastic dynamics: simulating the effects of turbulence on flexible structures," Computer Graphics Forum, vol. 16, no. 3, pp. C159-C164, 1997.

[55] T. Di Giacomo, S. Capo, and F. Faure, "An interactive forest," in Proceedings of the Eurographics Workshop on Computer Animation and Simulation, pp. 65-74, 2001.

[56] J. Weber and J. Penn, "Creation and rendering of realistic trees," in Proceedings of the 22nd Annual ACM Conference on Computer Graphics and Interactive Techniques (SIGGRAPH '95), pp. 119128, August 1995.

[57] P. Howlett and W. T. Hewitt, "Mass-spring simulation using adaptive non-active points," Computer Graphics Forum, vol. 17, no. 3, pp. C341-C353, 1998.

[58] D. Hutchinson, M. Preston, and T. Hewitt, "Adaptative refinement for mass-spring simulations," in Proceeding of the Eurographics Workshop on Animation and Simulation, pp. 149-159, 1996.

[59] S. Guerraz, F. Perbet, D. Raulo, F. Faure, and M. P. Cani, "A procedural approach to animate interactive natural sceneries," in Proceedings of the 16th International Conference on Computer Animation and Social Agents (CASA '03), pp. 73-78, 2003.

[60] X. Y. Hu, W. M. Tao, and Y. M. Guo, "Using FEM to predict tree motion in a wind field," Journal of Zhejiang University, vol. 9, no. 7, pp. 907-915, 2008.

[61] D. Sellier, T. Fourcaud, and P. Lac, "A finite element model for investigating effects of aerial architecture on tree oscillations," Tree Physiology, vol. 26, no. 6, pp. 799-806, 2006.

[62] X. Hu, H. Li, Y. Guo, and C. Song, "Dynamic finite element based animation of tree swing in the wind," Journal of Computer-Aided Design and Computer Graphics, vol. 19, no. 9, pp. 1166-1171, 2007. 

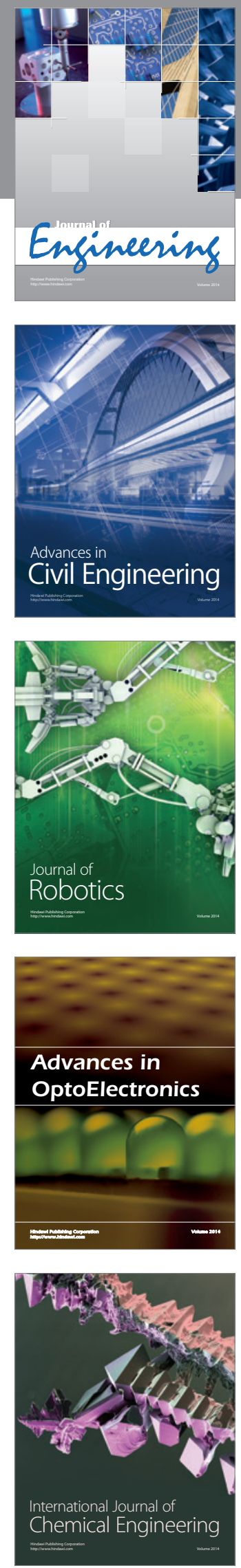

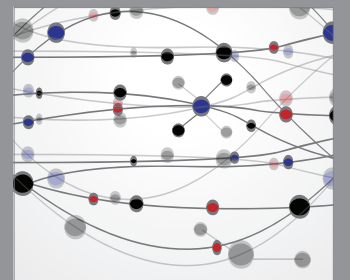

The Scientific World Journal
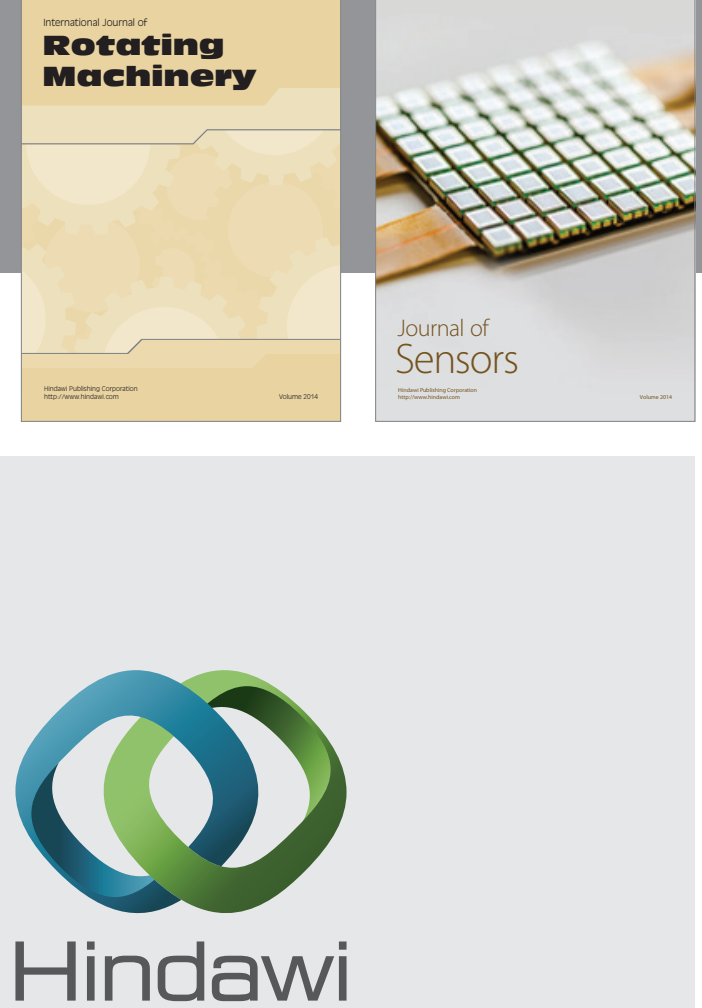

Submit your manuscripts at http://www.hindawi.com
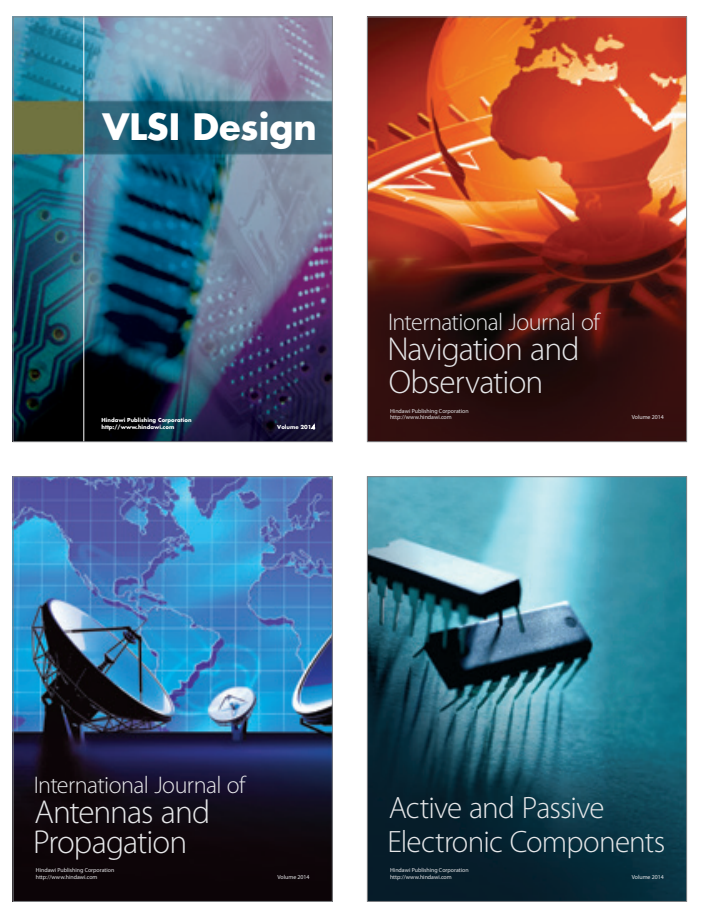
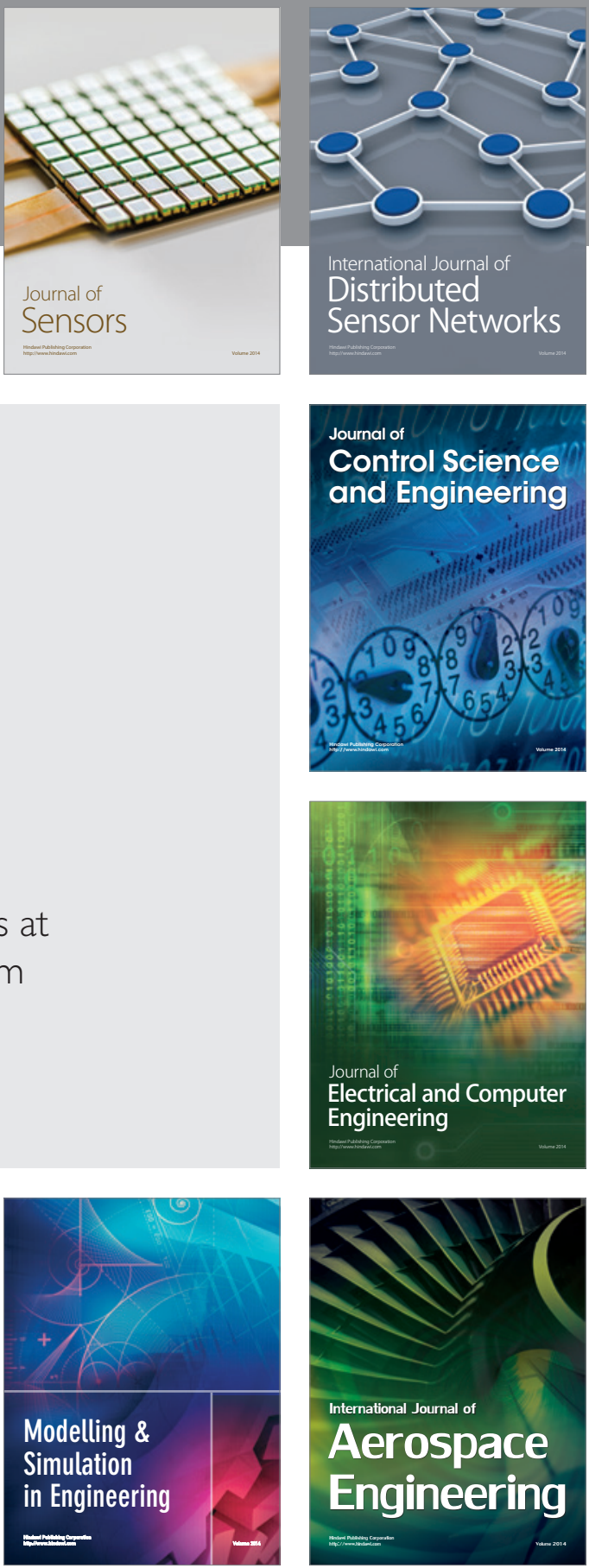

Journal of

Control Science

and Engineering
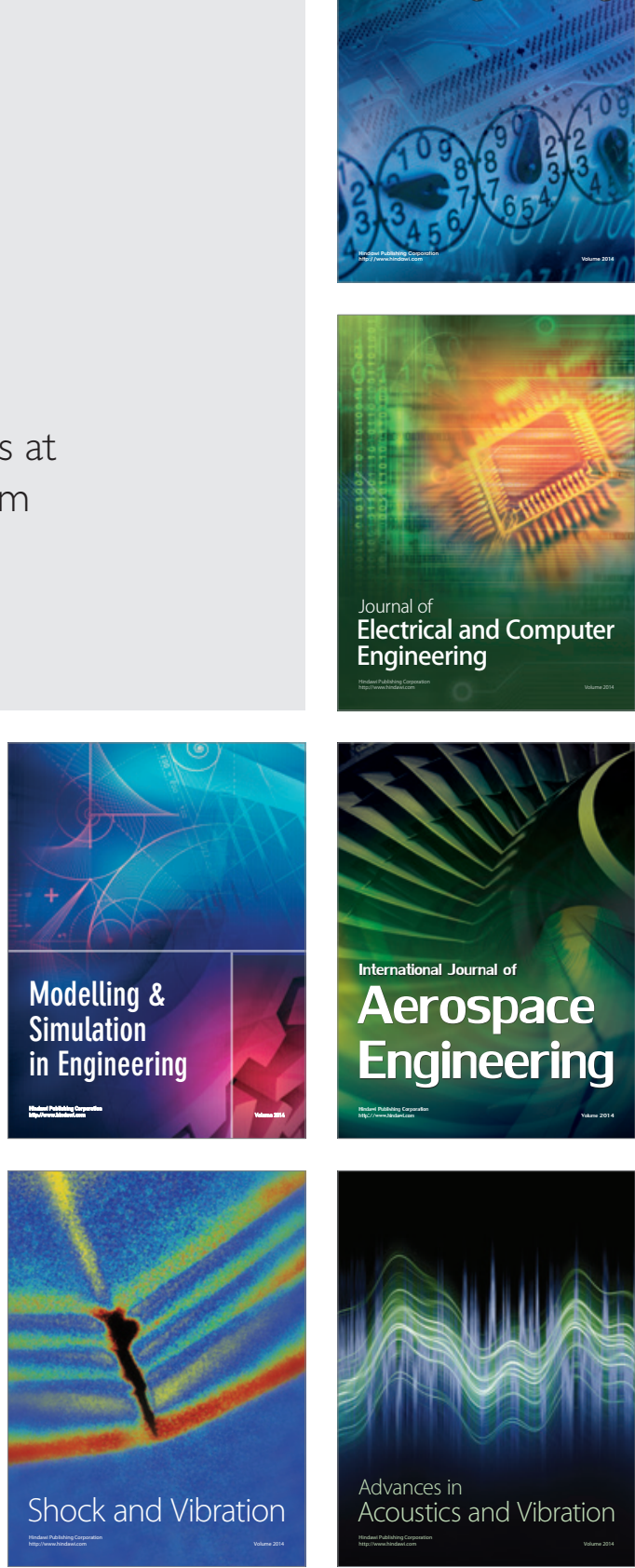